\title{
Gerontology
}

\section{Hyponatremia in Geriatric Inhospital Patients: Effects on Results of a Comprehensive Geriatric Assessment}

\author{
Markus Gosch ${ }^{a}$ Birgit Joosten-Gstrein ${ }^{a}$ Hans-Jürgen Heppner ${ }^{b}$ \\ Monika Lechleitner ${ }^{\text {a }}$

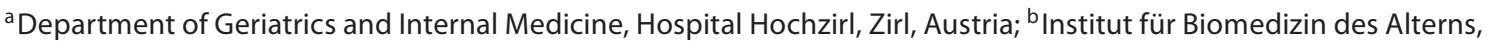 \\ Lehrstuhl Innere Medizin - Geriatrie, Universität Erlangen-Nürnberg, Nuremberg, Germany
}

\section{Key Words}

Hyponatremia • Geriatric assessment • Geriatric patients • Impairment

\begin{abstract}
Objectives: To study whether geriatric patients with mildto-moderate hyponatremia ( $\leq 131 \mathrm{mmol} / \mathrm{l}$ ) reveal different outcomes in structured tests for functional and cognitive impairments, depression and malnutrition compared to normonatremic patients. Design: Single-center, retrospective case control study. Setting: The study was conducted in a Geriatric Evaluation and Management Unit of a Department for Geriatrics and Internal Medicine. Methods and Participants: We included 2,880 elderly patients $(75.6 \%$ female, mean age $78.6 \pm 6.98$ years), consecutively admitted to the GEMU primarily or from another hospital or emergency department. Results were compared between a group of 129 patients with mild-to-moderate hyponatremia (118-131 mmol/l) and an age- and sex-matched control group of 129 patients with normal serum sodium values (>135 mmol/l). To assess functional and cognitive status, depression and malnutrition we used standardized tests of a geriatric assessment. Results: $16.7 \%(n=477)$ of the total $2,880$ patients were hyponatremic ( $\leq 135 \mathrm{mmol} / \mathrm{l}), 4.5 \%(\mathrm{n}=$ 129) revealed moderate hyponatremia. Compared to the
\end{abstract}

control group, these patients had significantly worse results in all tests of the Geriatric Assessment, including Activities of Daily Living, Mini Mental State Examination, Clock Completion Test, Geriatric Depression Score, Tinetti Mobility Test and the Timed Up\&Go Test and the Mini Nutritional Assessment. Comorbidities were assessed by the Charlson Comorbidity Index and the Cumulative Illness Rating Scale with no significant difference between the two groups. The hyponatremic patients received significantly more medications than the normonatremic control group, but we could not find a significant difference with respect to the use of a distinct single drug therapy. Conclusion: We were able to demonstrate that geriatric patients with mild-to-moderate hyponatremia revealed a significantly worse outcome in all standardized tests of the geriatric assessment compared to a normonatremic control group. Serum sodium levels should therefore be considered when interpreting common tests of geriatric assessment. Copyright $\odot 2012$ S. Karger AG, Basel

\section{Introduction}

Hypotonic hyponatremia is the most frequently observed electrolyte disorder in patients admitted to general hospitals [1]. According to the literature, the preva-

\section{KARGER \\ Fax +41613061234 \\ E-Mail karger@karger.ch}

www.karger.com
(C) 2012 S. Karger AG, Basel

0304-324X/12/0585-0430\$38.00/0

Accessible online at:

www.karger.com/ger
M. Gosch

Department of Geriatrics and Internal Medicine

Hospital Hochzirl

AT-6170 Zirl (Austria)

Tel. +435238 501, E-Mail markus.gosch@tilak.at 
lence of hyponatremia ranges from 9.4 to $15 \%$ in hospitalized patients $[2,3]$ and is particularly high among patients from nursing homes and all elderly persons [4]. The clinical symptoms are closely related to the severity and onset of hyponatremia.

The acute form is defined by an occurrence of clinical symptoms within $<48 \mathrm{~h}$ and is due to severe hyponatremia. A pronounced lowering of serum sodium levels increases the risk for intracranial hypertension and severe neurological symptoms [5].

In contrast to this critical situation, mild stable hyponatremia - defined as a serum sodium level of 120-132 $\mathrm{mmol} / \mathrm{l}$ - is generally considered to be clinically asymptomatic. However, several studies reported functional impairments in elderly patients under this condition. Renneborg et al. [1] determined the fall frequency in 122 patients with chronic hyponatremia and 244 controls, who were admitted to a medical emergency department. In this study, significantly more hyponatremic patients were admitted to hospital because of falls (21.3\%) than patients in the control group with normal sodium values $(5.3 \%)(\mathrm{p}<0.001)$. Gait and attention were also significantly impaired.

Decaux [6] applied attention and gait tests to assess physical function in 16 patients with asymptomatic hyponatremia (serum sodium $128 \pm 3 \mathrm{mmol} / \mathrm{l}$ ) resulting from inappropriate antidiuretic hormone secretion. Attention and gait were even more severely impaired in the patients than in age-matched volunteers who ingested $0.55 \mathrm{~g}$ alcohol per $\mathrm{kg}$ bodyweight before the performance of the tests.

Hyponatremia is also known as a risk factor for bone fractures in elderly [7]. The study by Sandhu et al. [8] demonstrated an association of lower sodium levels to the incidence of larger bone fractures in elderly patients. The frequency of falls was found to be not increased in patients with severe compared to mild hyponatremia [9].

A further aspect that has to be considered in the complex consequences of hyponatremia is the impaired nerve conduction velocity [10], which could affect balance regulation.

Overall, the prevention of falls in elderly has become a major public health interest. The elderly population is increasing, which is paralleled by an increase in the need for care facilities. Up to $5 \%$ of older people who fall require subsequent hospitalization. Gait and balance deficits, as well as cognitive impairments are known as independent fall risk factors, but also as clinical symptoms of hyponatremia $[6,11]$.

Hyponatremia in Geriatric Inhospital Patients
Drugs are a common cause of electrolyte abnormalities and one of the more common electrolyte abnormalities that may be drug induced is hyponatremia $[12,13]$. Drugs may cause mainly hyponatremia by affecting sodium and water homeostasis [12] like diuretics [14] or increasing the hypothalamic production of $\mathrm{ADH}$ [12] like antidepressants $[12,15]$, antipsychotic drugs [16], antiepileptic drugs [17-19] and opioide therapy [20].

The Comprehensvie Geriatric Assessment (CGA) is a standardized tool to screen for functional and cognitive disabilities, depression, delirium and malnutrition in elderly patients. It can be defined as 'a multidimensional, usually interdisciplinary, diagnostic process intended to determine an older person's medical, psychosocial, and functional capacity and problems with the objective of developing an overall plan for treatment and long-term follow-up' [21]. It has been used for many years to evaluate and address the complex and special needs of frail hospitalized older patients. The results of the CGA reveal a strong relation to the prognostic outcome and thus have a significant impact on the treatment of elderly patients. Until now, only few published data are available concerning the association of laboratory results and the outcome in the CGA.

Considering the importance of the CGA in the therapy plan for elderly patients and the discrepant results in the literature about the influence of laboratory results on functional disabilities we performed a retrospective analysis in a cohort of elderly patients undergoing a CGA. A main purpose was to study the association of mild-tomoderate hyponatremia to the results of standardized tests of the CGA.

\section{Methods}

Study Design

The present study is a retrospective case-control study. Data were collected between 2005/07/05 and 2008/01/07 at the Department of Geriatrics and Internal Medicine at the hospital of Hochzirl. The setting of our study corresponds to a Geriatric Evaluation and Management Unit (GEMU). The GEMU is 'a ward that admits frail older inpatients for a process of multidisciplinary assessment, review and therapy' [22].

Study Population

We included all patients who were consecutively admitted to our department primarily or from another hospital or emergency department. All patients underwent standard clinical and laboratory tests (including glucose, CRP, sodium, potassium, calcium, chloride, creatinine, urea, GOT, GPT, $\gamma$-GT, urine status) and a CGA on the second day after admission to our hospital. Patients who were not able to perform the tests of the Geriatric Assessment

Gerontology 2012;58:430-440 
Table 1. Patient characteristics: mean age, sex, mean serum sodium, recent surgical treatment, admission diagnoses (ICD-10 code, chapters) and length of stay

\begin{tabular}{|c|c|c|c|}
\hline Age & $78.6 \pm 7.0$ & $80.15 \pm 7.3$ & $80.15 \pm 7.3$ \\
\hline Females & $2,152(75.5 \%)$ & $102(79.1 \%)$ & $102(79.1 \%)$ \\
\hline Serum sodium, mmol/l & $138.39 \pm 3.7$ & $128 \pm 3.2$ & $139.35 \pm 2.5$ \\
\hline Recent surgical treatment - yes $^{a}$ & $1,299(45.6 \%)$ & $38(29.5 \%)$ & $26(20.2 \%)$ \\
\hline Diseases of the blood, blood-forming organs and immune system (D00-D90) & $34(1.2 \%)$ & $2(1.6 \%)$ & $5(3.9 \%)$ \\
\hline Endocrine, nutritional and metabolic diseases (E00-E90) & $169(5.9 \%)$ & $15(11.6 \%)$ & $10(7.8 \%)$ \\
\hline Mental and behavioral disorders (F00-F9) & $66(2.3 \%)$ & $5(3.9 \%)$ & $5(3.9 \%)$ \\
\hline Diseases of the nervous system (G00-G99) & $68(2.4 \%)$ & $4(3.1 \%)$ & $4(3.1 \%)$ \\
\hline Diseases of the ear and mastoid process (H00-H59) & $7(0.2 \%)$ & 0 & $1(0.8 \%)$ \\
\hline Diseases of the circulatory system (I00-I95) & $350(12.3 \%)$ & $18(14 \%)$ & $27(20.9 \%)$ \\
\hline Injury, poisoning and certain other consequences of external causes (S00-T98) & $591(20.7 \%)$ & $28(21.7 \%)$ & $14(10.9 \%)$ \\
\hline Length of stay, days & $18.64 \pm 8.6$ & $19.8 \pm 8.3$ & $16.8 \pm 8.9$ \\
\hline
\end{tabular}

Results are shown as absolute frequency and percentage, except age, sodium level and length of stay, which are shown as mean values \pm SD.

a Recent surgical treatment: 'yes' means that a patient underwent surgery within 4 weeks before the admission to our GEMU.

(such as patients in life-threatening conditions, patients with the diagnosis of a pronounced cognitive impairment and palliative patients) were not included in the evaluation.

During the study period a total of 2,880 elderly patients with a mean age $78.6 \pm 6.98$ years and a range of $65-102$ years could be evaluated. All patients were assigned to 6 age groups from 65 up to $>89$ years in order to determine an age influence on the prevalence of mild to moderate hyponatremia. The results obtained in hyponatremic patients were compared to an aged- and sex-matched control group $(n=129,79.1 \%$ female, $20.9 \%$ male, mean age $80.16 \pm 7.27$ years; range 65 to 98 years) with normal sodium values (between 136 and $145 \mathrm{mmol} / \mathrm{l}$ ).

\section{Definition of Hyponatremia}

According to literature, serum sodium levels from 131 to 118 $\mathrm{mmol} / \mathrm{l}$ are defined as mild and moderate hyponatremia. The lowest sodium level observed in our study population was 118 $\mathrm{mmol} / \mathrm{l}$. Our evaluation was based on the threshold level of $\leq 131 \mathrm{mmol} / 1$ for moderate hyponatremia in order to obtain an adequate number of patients for the study and to avoid a disproportionally high number of hyponatremic patients near to the standard value [3]. Patients with sodium levels higher than 145 $\mathrm{mmol} / \mathrm{l}$ - indicating an underlying disorder for hypernatremia with a need for further diagnostic procedures - were excluded from our evaluation.

\section{Data Collection}

Comorbidities and Further Results

To describe the diagnosis leading to hospital admission we used the International Classification of Diseases (ICD-10) (table 1). The high prevalence of diseases of the musculoskeletal system and connective tissue (chapter XIII, ICD-codes M00-M99) and of injuries, poisoning and certain other consequences of external causes (chapter XIX, ICD-codes S00-T98) is a consequence of the high percentage of admissions from orthopedic and trauma surgery units.

In order to evaluate and graduate the comorbidities in the subgroups we applied the Charlson Comorbidities Index (CCI) [23] as well as the Cumulative Illness Rating Scale (CIRS) [24, 25]. For calculation of the scores all comorbidities registered in the formerly documented and actual case histories were considered.

A nonvalidated questionnaire for urinary incontinence symptoms with 6 items was used to screen for urinary incontinence [26]. A patient with $\geq 1$ positive answer was classified as urinary incontinent. C-reactive protein values were determined in the blood samples obtained on the day of admission to our hospital.

\section{Causes of Hyponatremia}

We used the patients' charts to detect the causes of hyponatremia, looking for serumosmolarity and the diagnoses listed in the charts. We collected all diagnoses in connection with hyponatremia. 
Medication

Regarding the medication, the following substances were included (yes/no): nonsteroidal anti-inflammatory drugs, acetylsalicylic acid, thiazide diuretics, loop-diuretics, low potent neuroleptics, high potent neuroleptics, angiotensin-converting-enzyme (ACE) inhibitors, AT II blockers, tricyclic antidepressants, selective serotonin reuptake inhibitors (SSRI), selective serotonin and noradrenalin reuptake inhibitors (SSNI), carbamazepine, quiinolones, spironolactone, monoaminoxidase inhibitors, vasopressin, amiloride, opiates, indapamide, oxacarbazepine, valproic acid, methotrexate, chemotherapeutics, lamotrigine, chlorpropamide, tolbutamide, venlafaxine, amlodipine, amiodarone, lorcainide, propafenone, theophylline, proton pump inhibitors, telipressine, bupropione. The selection of these substances was based on a literature review performed on PubMed concerning medication and hyponatremia. Furthermore, total number of medications (NOM) and number of potentially hyponatremiainducing medications (NOHIM) reported by the patient at admission were both evaluated.

\section{Activities of Daily Living}

To assess the overall functional status we used the activities of daily living (ADL), described by the Barthel Index [27]. Data were provided by a nurse, who had observed the patient for $24 \mathrm{~h}$. The score ranges from 0 (dependent on assistance in daily activities (ADLs) such as bathing, eating and dressing) to 100 (independent) points. For the bivariate logistic regression model we defined patients with an ADL score of $<75$ points as relevant impairment in the activities of daily living.

\section{Cognition and Depression}

Two tests were applied to evaluate cognitive function, the Mini Mental State Examination (MMSE), a sufficient screening instrument for cognitive impairment, and the Clock Completion (CC) test, a sensitive test concerning practical cognitive skills $[28,29]$. Patients with a MMSE score $<28$ and a CC score $>3$ were defined as cognitive impaired. Because of the fact that elderly patients with serious diseases often suffer from symptoms of depression [30], we have applied the Geriatric Depression Scale (GDS) to screen for depressive disorders (short version) [31]. The short version contains 15 items, $>5$ positive answers are indicative for depression [31].

\section{Mobility and Risk of Falls}

The Tinetti Performance-Oriented Mobility Assessment (POMA), also called the Tinetti Mobility Test (TMT), and the Timed Up\&Go (TUG) are tools to determine impairments of mobility, gait and gait speed. The TMT predicts falls among elderly individuals; those scoring 20 to 24 of 28 on the TMT are at 'moderate' risk of falling, and individuals scoring $\leq 19$ are at 'high' risk of falling [32]. The TUG test of $\geq 20 \mathrm{~s}$ is a simple indicator of an older adult's impaired functional mobility [33]. It involves recording the time it takes the participant to stand up from a chair, walk $3 \mathrm{~m}$, turn, walk back to the chair and sit down.

\section{Nutrition}

The nutritional status of the patients was determined by the Mini Nutritional Assessment (MNA) [34]. Added MNA score allows one to identify elderly who have an adequate nutritional status, those who are at risk of malnutrition and those who are mal-
Table 2. Medications

\begin{tabular}{lccl}
\hline Substance & $\begin{array}{l}\text { Hyponatre- } \\
\text { mic group } \\
(\mathrm{n}=129)\end{array}$ & $\begin{array}{l}\text { Control } \\
\text { group } \\
(\mathrm{n}=129)\end{array}$ & $\begin{array}{l}\mathrm{p} \\
\text { value }^{\mathrm{a}}\end{array}$ \\
\hline NSAIDs incl. ASS & $62(48.1 \%)$ & $61(47.3 \%)$ & 0.901 \\
Diuretics & $88(68.2 \%)$ & $73(56.6 \%)$ & 0.054 \\
$\quad$ Thiazide diuretics & $52(40.3 \%)$ & $40(31 \%)$ & 0.119 \\
ACE inhibitors & $48(37.2 \%)$ & $51(39.5 \%)$ & 0.701 \\
AT II blockers & $20(15.5 \%)$ & $18(14 \%)$ & 0.725 \\
Neuroleptics & $15(11.6 \%)$ & $14(10.9 \%)$ & 0.844 \\
Antidepressant drugs ${ }^{\mathrm{d}}$ & $33(25.6 \%)$ & $25(19.4 \%)$ & 0.233 \\
$\quad$ SSRI & $25(19.4 \%)$ & $21(16.3 \%)$ & 0.515 \\
Antiepileptic drugs ${ }^{\mathrm{e}}$ & $3(2.3 \%)$ & $1(0.8 \%)$ & 0.314 \\
Quinolones & $8(6.2 \%)$ & $2(1.6 \%)$ & 0.053 \\
Opioids & $22(18.6 \%)$ & $12(9.3 \%)$ & 0.066 \\
Methotrexate & 0 & $3(2.3 \%)$ & 0.083 \\
Amlodipine & $20(15.4 \%)$ & $18(14 \%)$ & 0.725 \\
Amidarone & $1(0.8 \%)$ & $1(0.8 \%)$ & 1 \\
Theophylline & $8(6.2 \%)$ & $7(5.4 \%)$ & 0.790 \\
Proton pump inhibitors & $60(46.5 \%)$ & $54(41.9 \%)$ & 0.452 \\
NOM & $8.4 \pm 3.8$ & $6.9 \pm 3.6$ & 0.002 \\
NOHIMg & $3.33 \pm 1.8$ & $2.85 \pm 1.73$ & 0.034 \\
\hline
\end{tabular}

${ }^{a} p$ value was calculated by using the Pearson $\chi^{2}$ test (for categorical data) and the Mann-Whitney $U$ test (for comparing mean values).

b Diuretics include thiazides, loop diuretics, spironolactone, amiloride and indapamide (thiazides are also shown separately).

${ }^{c}$ Neuroleptics include low-potent, high-potent and atypical neuroleptics.

${ }^{d}$ Antidepressant drugs include tricyclic antidepressants, SSRI, SSNI, MAO inhibitors and venlafaxine (SSRI are also shown separately).

e Antiepileptic drugs include carbamazepine, valproic acid, and lamotrigine.

Vasopressin, oxacarbazepine, lorcainide, propafenone, telipressine and bupropione were not used in our patient collective. Results are shown as absolute frequency and percentage, number of medications as mean value and SD.

$\mathrm{f}$ Total number of medications.

${ }^{\mathrm{g}}$ Number of potentially hyponatremia-inducing medications.

nourished. For our bivariate logistic regression we compared the patients with a MNA score $<18$ points.

All of the specific assessment tests were performed under the control of a nurse specialized for the geriatric test program.

\section{Statistical Analysis}

The SPSS version 20.0 (2011) was applied for statistical analysis. Metric scaled data are reported as arithmetic mean \pm SD and categorical data as absolute frequency and percentage distribution. Non parametric statistics (Mann-Whitney U test) were used since normality assumptions were not met for most of the outcome variables. Group effect and main condition effects were tested for significance by the Mann-Whitney $U$ test and Wilcoxon 
Fig. 1. Patient age distribution and frequency of hyponatremia (\%) for all age groups.

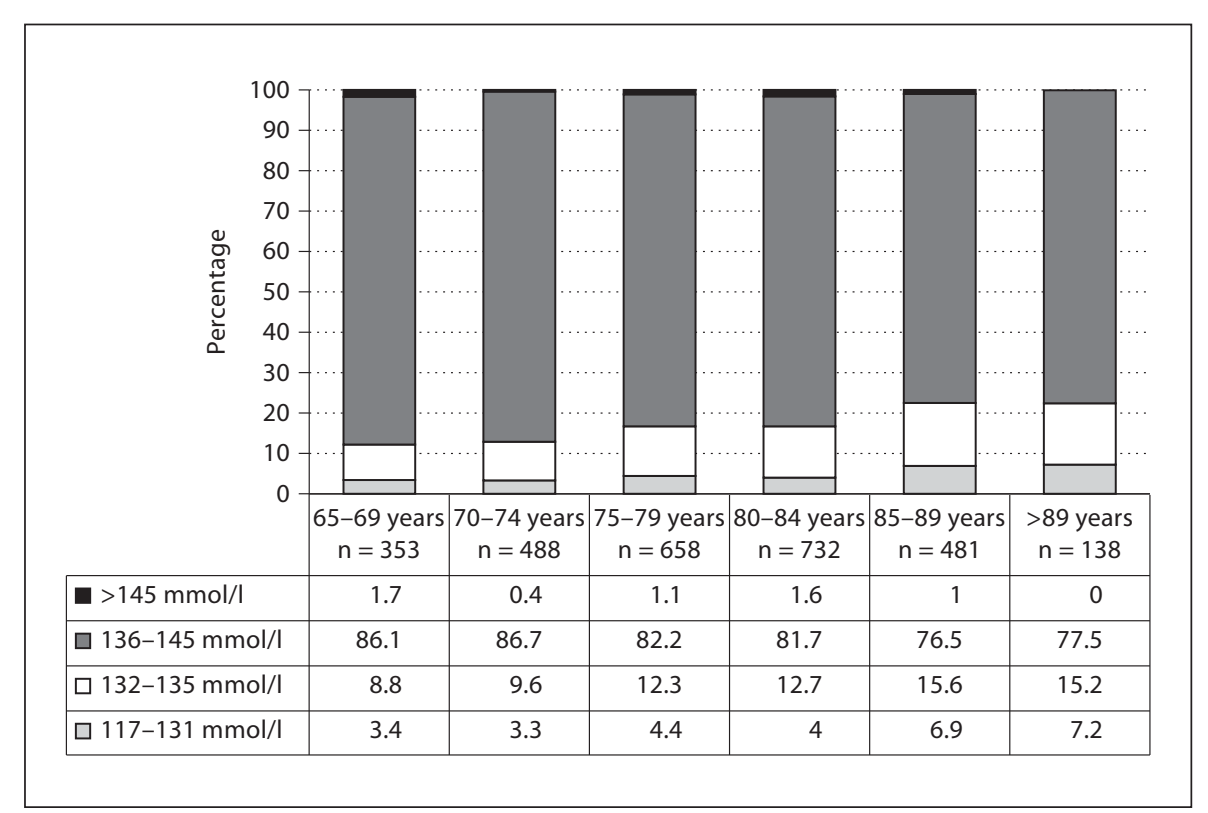

signed rank test. The $\chi^{2}$ test for independence was used to determine a possible relationship between two categorical variables. The significance level was defined by $\mathrm{p}<0.05$.

Multivariate logistic regression analyses were performed to identify factors associated with worse results in the CGA. The dependent variables for these analyses were the different tests of the CGA (ADL, MMSE, GDS, TMT, TUG, MNA, urinary incontinence). The independent variables were hyponatremia, age, recent surgical therapy, all items of the CCI and the CIRS, their total scores and the medication (as shown in table 2). Bivariate analyses were based on logistic regression to generate odds ratios (OR) and 95\% CI. Independent variables were retained in the models when $\mathrm{p}<0.150$.

\section{Results}

Overall a total of 2,880 patients with a mean age of $78.6 \pm 6.98$ years (female $75.6 \%$, male $24.4 \%$ ) were included in our evaluation. 477 (16.7\%) patients were hyponatremic (serum sodium $\leq 135 \mathrm{mmol} / \mathrm{l}), 129(4.5 \%)$ revealed mild or moderate hyponatremia (serum sodium 131-118 mmol/l). Thirty-two (1.1\%) of 2,880 patients were hypernatremic. Patients with mild-to-moderate hyponatremia (female $79.1 \%$, male $20.9 \%$ ) had a mean age of $80.16 \pm 7.32$ years and a mean sodium value of 127.98 $\mathrm{mmol} / \mathrm{l}$. The mean age of the normonatremic control group was $80.16 \pm 7.32$ years (female $79.1 \%$, male $20.9 \%$ ) with a mean sodium value of $138 \mathrm{mmol} / \mathrm{l}$. The prevalence of hyponatremia revealed peak values in the oldest age groups $\geq 85$ years (fig. 1). The increase in the prevalence of hyponatremia among these older age groups is significant $(\mathrm{p}=0.004)$ (table 3 shows the results of the CGA for each age group).

\section{Comorbidities and Further Results}

The calculated CCI revealed no significant difference $(\mathrm{p}=0.208)$ between the hyponatremic patients $(2.33 \pm$ $1.93)$ and the control group $(2.01 \pm 1.97)$. Patients with hyponatremia had a higher prevalence of peripheral vascular disease (14 vs. $2.3 \%$; $\mathrm{p}=0.001$ ), chronic lung disease (25.6 vs. $12.4 \% ; \mathrm{p}=0.007$ ) and connective tissue disease ( 1.6 vs. $8.5 \% ; \mathrm{p}=0.010)$. Using the CIRS, we could not find a significant difference in the total score between the two groups. Respiratory problems were significantly more frequent in the hyponatremic than in the control group (table 4). Hyponatremic patients revealed symptoms of urinary incontinence more frequently than the control group ( 60.9 vs. $48.3 \%$; $\mathrm{p}=0.016$ ). This finding was supported by the results of multivariate analysis, demonstrating a significantly a higher risk for urinary incontinence in hyponatremic patients $(\mathrm{p}=0.003, \mathrm{OR} 2.69,95 \%$ CI 1.39-5.20). With respect to laboratory results, the mean C-reactive protein was significantly higher in patients with hyponatremia $(2.39 \pm 3.24 \mathrm{mmol} / \mathrm{l}) \mathrm{com}-$ pared to the control group $(1.26 \pm 2.14 \mathrm{mmol} / \mathrm{l}$ in $\mathrm{p}<$ 0.001).

Patients with hyponatremia needed a significantly longer hospital treatment (19.8 \pm 8.3 days) than those in the control group $(16.8 \pm 8.9$ days; $p=0.002)$. 
Table 3. Results of the CGA for each age group and total

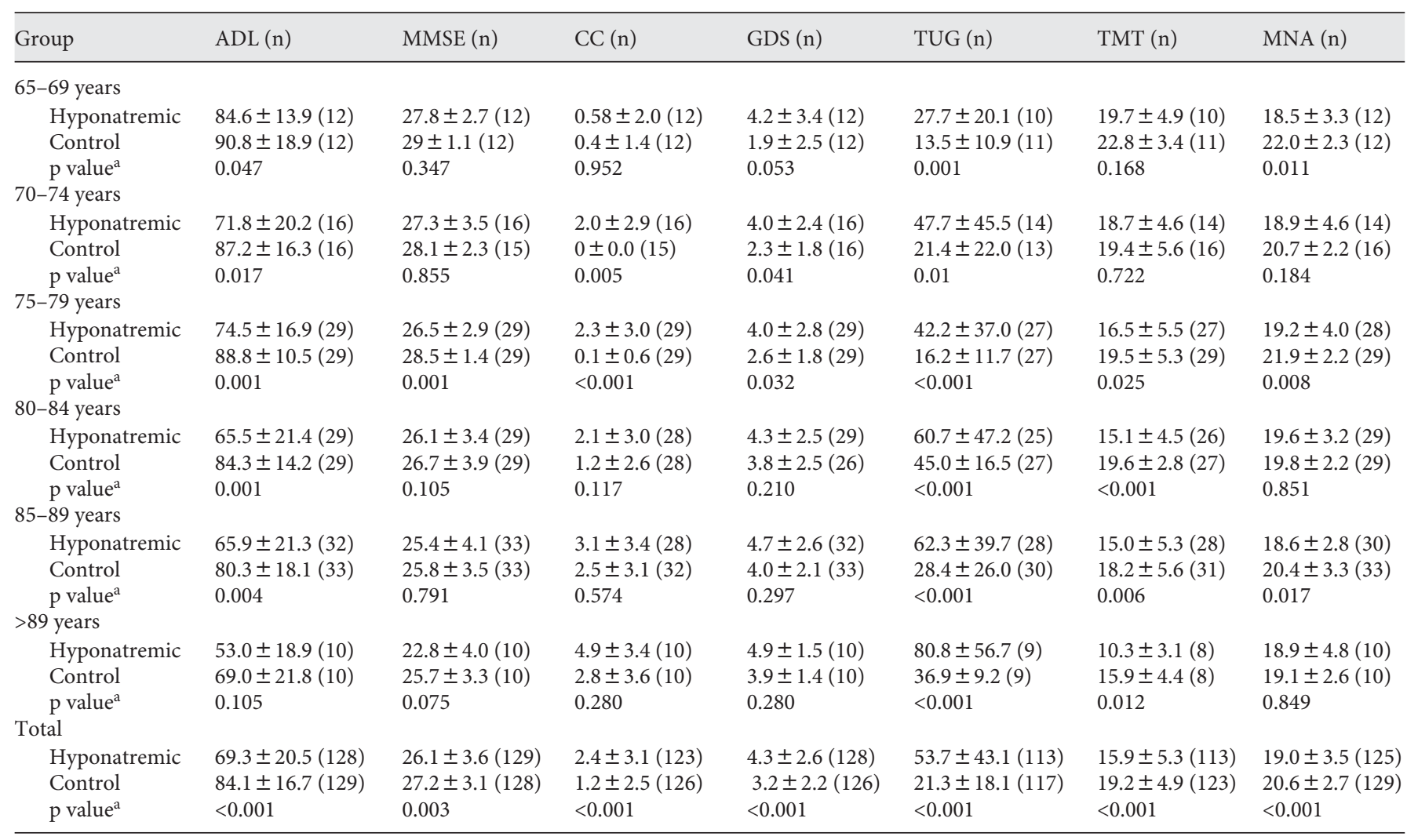

Results are shown as mean values \pm SD.

a $\mathrm{p}$ value was calculated by using the Mann-Whitney $U$ test comparing hyponatremic patients and controls in each age group.

\section{Causes of Hyponatremia}

According to the retrospective study design, the information about the probable causes of hyponatremia was based on the case histories. Serum osmolarity was available from 8 patients $(6.3 \%), 6(4.7 \%)$ of these measurements were found to be hypo-osmolar and $2(1.6 \%)$ normo-osmolar. For 99 patients $(76.8 \%)$ the diagnosis at discharge from hospital offered no underlying disease responsible for hyponatremia.

Adverse drug reaction (ADR) was described as the most common reason for hyponatremia (20 cases, $15.5 \%$ ). Further cases of hyponatremia were related with heart failure in $4(3.1 \%)$, severe liver disease in $1(0.8 \%)$, cancer in $2(1.6 \%)$, adrenal insufficiency in $2(1.6 \%)$ and syndrome of inappropriate antidiuretic hormone secretion in $1(0.8 \%)$.

\section{Medication}

The NOM taken by a patient was significantly higher in the hyponatremic group $(8.4 \pm 3.8)$ than in the control group $(6.9 \pm 3.6)(p=0.002)$. Also, the NOHIM taken by the patients of the hyponatremic group was significantly higher than in the control group $(3.33 \pm 1.8$ vs. $2.85 \pm$ 1.73) $(\mathrm{p}=0.034)$. However, there were no significant differences regarding one single drug or medication group (like diuretics, neuroleptics, antidepressive and antiepileptic drugs). All details are shown in table 5. In the multivariate analyses the effect of the different medications on the CGA was small and not significant.

\section{Overall Function - ADL}

ADL scores of 128 (99.2\%) hyponatremic and 129 (100\%) control group patients were available. The mean ADL score of the hyponatremic group was $69.26 \pm 20.52$ points compared to $87.56 \pm 13.08$ in the control group $(\mathrm{p}<0.001)$. Age $(\mathrm{p}<0.001)$, increased $\mathrm{C}$-reactive protein levels $(p<0.001)$, recent surgical treatment $(p=0.024)$, heart failure $(\mathrm{p}=0.012)$, cerebrovascular disease $(\mathrm{p}=$ $0.010)$, hemiplegia $(\mathrm{p}=0.020)$, PPI $(\mathrm{p}=0.016)$, peripheral vascular diseases $(\mathrm{p}=0.024)$ and CIRS score $(\mathrm{p}=0.003)$ 


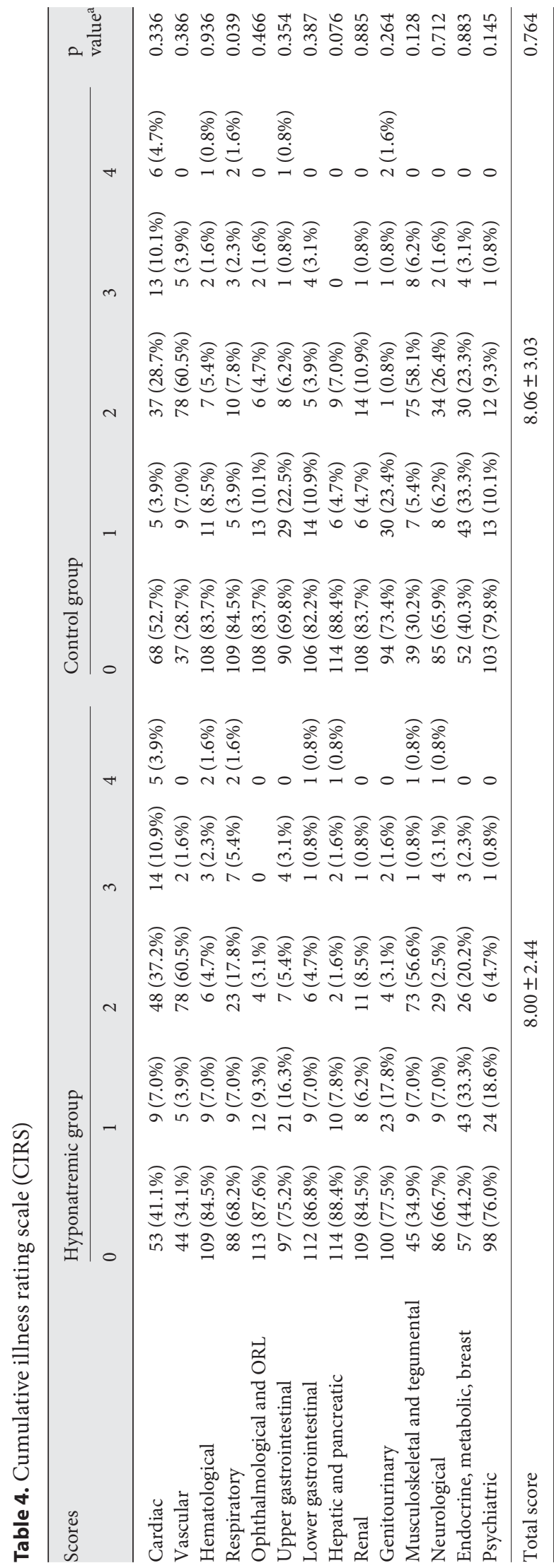

revealed a significant positive correlation with more pronounced impairments in the activities of daily living. In the multivariate analysis only hyponatremia $(\mathrm{p}<0.001$, OR 5.27, 95\% CI 2.62-10.60) and age ( $<<0.001$, OR 1.1, 95\% CI 1.04-1.16) remained significantly associated with an ADL score $<75$ points. We found no correlations with the medication to the ADL score, except the fact that patients under ACE-inhibitor therapy revealed a more favorable outcome $(\mathrm{p}=0.017$, OR $0.40,95 \%$ CI $0.19-0.85)$.

\section{Cognitive Function and Depression}

The MMSE scores of 129 (100\%) hyponatremic patients were analyzed and the mean MMSE value was $26.05 \pm 3.64$ points. In the control group data from 128 (99.2\%) patients were available. The mean MMSE score was significantly higher in the control group (27.18 \pm 3.15 points; $p=0.003$ ) compared to the hyponatremic patients. With a cut-off value of $>27$ in the MMSE test, 64 $(49.6 \%)$ patients in the hyponatremic group revealed a normal cognitive function compared to $84(65.6 \%)$ in the control group $(p=0.009)$. A significant positive correlation of the MMSE results was found with age $(p<0.001)$, C-reactive protein values $(\mathrm{p}=0.045)$, dementia $(\mathrm{p}=$ $0.001)$, cerebrovascular diseases $(p=0.036)$, hemiplegia $(\mathrm{p}=0.042)$, musculoskeletal and tegumental diseases $(\mathrm{p}=0.045)$ and opioids $(\mathrm{p}=0.043)$. In multivariate analyses hyponatremia ( $\mathrm{p}=0.036$, OR $1.96,95 \%$ CI $1.05-$ 3.68), dementia ( $\mathrm{p}=0.002$, OR 10.01, 95\% CI 2.26-44.32) and age $(\mathrm{p}<0.001$, OR $1.10,95 \%$ CI 1.05-1.16) could be demonstrated as significant predictors for a MMSE score $<28$. The CIRS item musculoskeletal and tegumental diseases ( $\mathrm{p}=0.018$, OR $0.70,95 \%$ CI $0.52-0.94)$ and the CCI item ulcer disease ( $\mathrm{p}=0.040$, OR $0.22,95 \%$ CI $0.05-0.935$ ) were statistically related to a better result of the MMSE test.

The CC test could be performed in 123 (95.4\%) hyponatremic patients and in 127 (98.5\%) of the controls. The mean score was $2.44 \pm 3.13$ points in hyponatremic patients compared to $1.17 \pm 2.52$ points in the control group. In the hyponatremic group only 80 patients (65\%) were able to follow the test in a correct way, compared to 105 (82.7\%) of the controls (cut-off of $<4$ points). The CC test results also revealed a significant positive association with age $(\mathrm{p}<0.001)$, rheumatism $(\mathrm{p}=0.037)$, hemiplegia $(\mathrm{p}=0.003)$, peripheral vascular $(\mathrm{p}=0.035)$ and endocrine, metabolic and breast diseases $(\mathrm{p}=0.022)$. After logistic regression only hyponatremia $(\mathrm{p}=0.014$, OR $2.40,95 \%$ CI 1.19-4.82) and age ( $\mathrm{p}<0.001$, OR 1.10, 95\% CI $1.05-$ 1.16) had significant variables. 
Table 5. Adjusted ORs of hyponatremia to predict worse outcomes in CGA

\begin{tabular}{lccc}
\hline & OR & $95 \%$ CI & p value \\
\hline Activities of Daily Living & $5.27^{\mathrm{a}}$ & $2.62-10.60$ & $<0.001$ \\
Mini Mental State Examination & $1.96^{\mathrm{b}}$ & $1.05-4.19$ & 0.036 \\
Clock Completion & $2.40^{\mathrm{c}}$ & $1.19-4.82$ & 0.014 \\
Geriatric Depression Score & $2.57^{\mathrm{d}}$ & $1.19-5.55$ & 0.017 \\
Tinetti Mobility Test & $3.73^{\mathrm{e}}$ & $1.88-7.39$ & $<0.001$ \\
Timed Up\&Go & $29.30^{\mathrm{f}}$ & $10.90-78.78$ & $<0.001$ \\
Mini Nutritional Assessment & $2.63^{\mathrm{g}}$ & $1.32-5.24$ & 0.006 \\
Urinary incontinence & $1.88^{\mathrm{h}}$ & $1.05-3.35$ & 0.033 \\
\hline
\end{tabular}

a Adjusted for age, C-reactive protein, CCI, recent surgical treatment, heart failure, dementia, cerebrovascular diseases, hemiplegia, CIRS, cardiac, vascular, hematological, genitourinary and neurological diseases, diuretics, ace inhibitors, quinolones, amlodipine PPIs, NOM, NOHIM listed in table 2.

${ }^{\mathrm{b}}$ Adjusted for age, C-reactive protein, CCI, heart failure, dementia, cerebrovascular diseases, ulcer disease, mild liver disease, hemiplegia, hepatic and pancreatic, renal, musculoskeletal and tegumental, neurological diseases, diuretics, sartane and opioids.

${ }^{c}$ Adjusted for age, C-reactive protein, rheumatism, mild liver disease, diabetes without complications, hemiplegia, renal failure, vascular, respiratory, renal, musculoskeletal and tegumental, endocrine, metabolic and breast diseases.

d Adjusted for myocardial infarction, cerebrovascular diseases, diabetes without complications, renal failure, lymphoma, ophthalmological and ORL, psychiatric diseases, NSAIDs, SSRI, antidepressant drugs, quinolones, opioids, NOM and NOHIM listed in table 2 .

e Adjusted for age, CIRS, C-reactive protein, recent surgical treatment, diabetes without complications, musculoskeletal and tegumental diseases, psychiatric diseases, opioids, methotrexate and PPIs.

${ }^{\mathrm{f}}$ Adjusted for age, C-reactive protein, recent surgical treatment, diabetes without complications, musculoskeletal and tegumental diseases, NOM, NOHIM listed in table 2, opioids, PPIs.

g Adjusted for peripheral arterial disease, dementia, hemiplegia, vascular diseases, ACE inhibitors, antidepressive drugs and chinolones.

$\mathrm{h}$ Adjusted for total number of medications, recent surgical treatment and chronic lung diseases.

Also, the GDS revealed a significant difference between hyponatremic and normonatremic patients. We evaluated a GDS score from 128 (98.5\%) patients in the hyponatremic group and 127 (98.5\%) in the control group. The mean GDS score was $4.34 \pm 2.6$ points in the hyponatremic group and $3.2 \pm 2.2$ points in the control group $(\mathrm{p}<0.001)$. Using a cut-off of $>5$ points, 34 hyponatremic patients revealed symptoms of depression, but only 15 patients of the control group $(\mathrm{p}=0.003)$. Diabetes without complications $(p=0.048)$, psychiatric diseases $(p=0.007)$, the NOM $(\mathrm{p}=0.009)$ as well as all antidepressive drugs $(p=0.028)$ and SSRIs $(p=0.034)$ were significantly related to the GDS score. After multivariate analysis only hyponatremia ( $\mathrm{p}=0.017$, OR $2.57,95 \%$ CI $1.19-5.55)$ and psychiatric diseases $(\mathrm{p}=0.021$, OR $1.86,95 \%$ CI $1.10-3.15)$ remained as significant variables associated with the GDS.

\section{Mobility}

$113(87.6 \%)$ patients in the hyponatremic group were able to perform the TUG, as compared to 117 (90.7\%) in the control group. Patients who are not able to perform these mobility tests are classified as immobile without the help of another person. Fourteen patients in the hyponatremic group, but only 6 in control group were found to be immobile. The mean time for the TUG test was 53.73 $\pm 43.15 \mathrm{~s}$ in the hyponatremic group compared to 21.3 $\pm 7.99 \mathrm{~s}$ in the control group $(\mathrm{p}<0.001)$. Age $(\mathrm{p}<0.001)$, $\mathrm{C}$-reactive protein $(\mathrm{p}<0.001)$, recent surgical treatment $(p<0.001)$, opioids $(p=0.008)$ and the NOM $(p=0.006)$ had a significant negative effect on the results of the TUG. Patients with diabetes without complications revealed better results in the mobility test $(\mathrm{p}=0.014)$. Multivariate analysis demonstrated that hyponatremia had the strongest impact on the TUG outcome $(\mathrm{p}<0.001$, OR 32.77, 95\% CI 11.36-94.52). Age ( $\mathrm{p}<0.001$, OR 1.18, 95\% CI $1.10-1.26)$, C-reactive protein ( $\mathrm{p}=0.021$, OR $1.45,95 \%$ CI $1.06-1.98)$ and recent surgical treatment $(\mathrm{p}=0.008$, OR $4.94,95 \%$ CI 1.51-16.19) remained also significantly negatively correlated with the results of the TUG. Patients with psychiatric diseases revealed better results $(\mathrm{p}=$ 0.020 , OR $0.41,95 \%$ CI 0.19-0.87), whereas patients treated with an antidepressive medication had a significant higher risk for a TUG $\geq 20 \mathrm{~s}(\mathrm{p}=0.046$, OR $3.11,95 \%$ CI 1.02-9.92).

We could also observe a significant difference between hyponatremic and normonatremic patients in the performance of the TMT. In the hyponatremic group, $11 \mathrm{pa}-$ tients were not able to complete the test, which could be completed by all patients in the control group. The mean TMT score was $15.9 \pm 5.86$ in patients with hyponatremia compared to $19.2 \pm 4.23$ in the control group $(\mathrm{p}<$ 0.001 ). In accordance to the TUG test results diabetic patients without complications revealed a more favourable outcome $(\mathrm{p}=0.038)$. Age $(\mathrm{p}<0.001)$, C-reactive protein $(p=0.003)$, recent surgical treatment $(p=0.004)$, CIRS $(\mathrm{p}=0.049)$, musculoskeletal and tegumental diseases $(\mathrm{p}=0.005)$, PPIs $(\mathrm{p}=0.015)$ and the NOM $(\mathrm{p}=0.020)$ were significantly correlated with worse outcome in the TMT. After logistic regression hyponatremia $(\mathrm{p}=0.001$, OR 
3.34, 95\% CI 1.65-6.77), age ( $\mathrm{p}<0.001$, OR 1.11, 95\% CI 1.06-1.17) and musculoskeletal and tegumental diseases ( $\mathrm{p}=0.003$, OR $1.74,95 \%$ CI 1.21-2.49) effect remained as significant variables for the TMT.

\section{Nutrition}

We were able to evaluate the MNA score of 125 (96.8\%) hyponatremic patients and $129(100 \%)$ in the control group. The mean MNA score was $19.0 \pm 3.5$ points compared to a mean MNA of $20.6 \pm 2.64$ in the control group $(\mathrm{p}<0.001)$. Peripheral arterial disease $(\mathrm{p}=0.012)$, dementia $(\mathrm{p}=0.004)$, hemiplegia $(\mathrm{p}=0.001)$ and antidepressive medication $(\mathrm{p}=0.032)$ were also associated with a MNA score $<18$ points. The use of ACE-inhibitors was significantly related to a higher MNA score $(p=0.031)$. In multivariate analysis only hyponatremia $(\mathrm{p}=0.002$, OR 3.43 , $95 \%$ CI 1.56-7.56) and dementia ( $\mathrm{p}=0.026$, OR $4.20,95 \%$ CI 1.10-14.81) showed a significant correlation with a low MNA score. BMI scores of the hyponatremic group were significantly lower than in the control group (BMI 23.8 \pm 4.7 compared to $26.4 \pm 5.0, \mathrm{p}<0.001$ ).

\section{Discussion}

The results of our present evaluation demonstrate that elderly patients with mild-to-moderate hyponatremia compared to a control group with normal sodium levels - had a significantly worse outcome in cognitive and functional tests of the CGA. While previously published studies reported primarily the association of hyponatremia with a certain aspect of the geriatric assessment, especially mobility tests and the risk of falls $[1,6,7]$, we have applied a very comprehensive test battery including mobility, cognitive function and screening for depression and malnutrition. Significantly more patients with hyponatremia showed a worse outcome or either were not able to perform the cognitive and mobility tests. The mean ADL score in the hyponatremic patients was $69.3 \mathrm{com}$ pared to 87.6 in the control group ( $\mathrm{p}<0.001)$. The severity of impairments found in our evaluation for patients with mild-to-moderate hyponatremia is thus relevant for daily living. Even in the multivariate analyses hyponatremia remained an independent predictor. Age had also a quite strong effect on the results. Other variables like comorbidities and medication showed only a weak and inconsistent association.

According to the literature, mild-to-moderate hyponatremia corresponded to a serum sodium level of $\geq 115$ $\mathrm{mmol} / \mathrm{l}$. The prevalence of hyponatremia defined by so- dium values $\leq 135 \mathrm{mmol} / \mathrm{l}$ was $16.7 \%$ in our study population, which is in accordance to published studies [2, 3], $4.5 \%$ of all patients revealed serum sodium values between $\leq 131$ and $\geq 118 \mathrm{mmol}$. The prevalence of hyponatremia increased with increasing age, which might be due to an increase in comorbidities and the impaired renal function as well as a higher risk of malnutrition. The factors responsible for the age-related changes in renal sodium handling are still incompletely defined. Glomerular filtration rate, physical factors determined in part by hemodynamics, the renin-aldosterone system and the atrial natriuretic peptide, are all determinants of renal sodium handling and each has been demonstrated to vary significantly with age [3]. Due to our retrospective study design we used serum creatinine as the only parameter to screen for renal failure, despite its well-known poor correlation with glomerular filtration rate [36], especially in elderly people. In our evaluation the hyponatremic patients did not differ relating to the serum creatinine levels.

Furthermore, our results show that the relevance of especially mild - hyponatremia in clinical practice is underestimated. Specific laboratory tests were only done in $6.3 \%$ of the cases. A discharge diagnose in relationship to hyponatremia was found in one of four patients. These facts explain why we were not able to detect the reasons of hyponatremia in a sufficient way. Remarkable was the fact that ADRs were listed as the most common causes. This observation is in contrast to our findings concerning the medication. Some medications (e.g. diuretics) are taken more often by hyponatremic patients, but these differences were not significant. Our results are comparable with further studies $[37,38]$. However, hyponatremic patients received significantly more medications, particularly more drugs that are known to cause hyponatremia.

There are few studies evaluating the role of drugs in the prevalence of hyponatremia in geriatric patients. Rosholm et al. [37] investigated the prevalence of hyponatremia in 332 very old nonhospitalised people focusing on drug use. In total, 19 persons had hyponatremia. Of the drugs generally known to cause hyponatremia, only omeprazole and oral antidiabetic agents were associated with significantly lower medium sodium concentrations. The only drug class associated with clinically relevant hyponatremia was thiazide diuretics, which was used by significantly more patients with hyponatremia. Severe hyponatremia was rarely seen and the authors concluded that drugs have only a limited influence on serum sodium concentration. Passare et al. [38] investigated the prevalence of hyponatremia and association with drug 
use in elderly people, coming to the conclusion that the prevalence of hyponatremia increases with age and that there is an association with carbamazepine, laxatives, diuretics and ACE-inhibitors.

On the one hand, the impact of medication to induce hyponatremia might be overestimated, on the other hand we have to be careful to interpret our results in regard to ADRs. ADRs are a well-known phenomenon but their incidence is quite rare and our study population might be too small to detect ADRs in a significant way. The fact that the total number of prescribed drugs is the most important risk factor of ADRs is already well described in the literature [39].

Although the CCI did not differ significantly between patients with hyponatremia and the control group, hyponatremic patients were suffering more frequently from peripheral vascular disease, chronic lung disease and connective tissue disease. The CCI is a valid score to assess the overall comorbidities, but it does not estimate the severity of a single disease, for example in cases of heart failure. The CIRS evaluates not only the prevalence of different diseases in one individual; furthermore, it describes the problems caused by one or more diseases in one organ system. In our study we found in nearly all items no significant differences, with the exception of respiratory problems. Greenberg and Lehrich [40] found a higher mortality in hyponatremic patients suffering from cardiovascular, oncology and orthopedic diseases, but it remained unclear if hyponatremia itself leads to the higher mortality rate or if hyponatremia was just a marker for the severity of the diseases.

Our study reveals some limitations, especially with respect to the number of patients, and the fact, that only hospitalized patients were evaluated. No long-term observation data were available from the patients, in order to determine the influence of a normalized serum sodium level on functional outcome. Also the retrospective design which hinders the evaluation of causality of hyponatremia must be mentioned as limitation.

Despite these considerations we think that the results of our study are of clinical importance, regarding the interpretation of the CGA and consequences on $\mathrm{ADL}$ as well as the diagnostic and therapeutic procedures for elderly patients. Further studies are necessary to search for the short- and long-term outcome of elderly hyponatremic patients, particularly if it is possible to treat hyponatremia in an effective way.

\section{Disclosure Statement}

The authors declare no conflicts of interest. H.-J.H. is scholarship holder of the Forschungskolleg Geriatrie, Robert-BoschFoundation, Germany, as an unrestricted grant.

\section{References}

1 Renneboog B, Musch W, Vandemergel X, et al: Mild chronic hyponatremia is associated with falls, unsteadiness and attention deficits. Am J Med 2006;119:71.e1-e8.

2 Passare G, Viitanen M, Törring O, et al: Sodium and potassium disturbances in the elderly: prevalence and association with drug use. Clin Drug Invest 2004;24:535-544.

3 Vaidya C, Ho W, Freda BJ: Management of hyponatremia: providing treatment and avoiding harm. Cleve Clin J Med 2010;77: 715-726.

4 Thompson CJ: Hyponatremia: new association and new treatments. Eur J Endocrinol 2010;162(suppl 1):S1-S3.

5 Adrogue HJ, Madias NE: Hyponatremia. N Engl J Med 2000;342:1581-1589.

6 Decaux G: Is asymptomatic hyponatremia really asymptomatic? Am J Med 2006; 119:S79-S82.

7 Kinsella S, Moran S, Sullivan MO, et al: Hyponatremia independent of osteoporosis is associated with fracture occurrence. Clin J Am Soc Nephrol 2010;5:275-280.

Hyponatremia in Geriatric Inhospital Patients
8 Sandhu HS, Gilles E, DeVita MV, et al: Hyponatremia associated with large-bone fracture in elderly patients. Int Urol Nephrol 2009;41:733-737.

9 Hoorn EJ, van der Lubbe N, Zietse R: SIADH and hyponatremia: why does it matter. NDT Plus 2009;2(suppl 3):iii5-iiil1.

10 Arányi Z, Kovács T, Szirmai I, et al: Reversible nerve conduction slowing in hyponatremia. J Neurol 2004;251:1532-1533.

11 American Geriatrics Society, British Geriatrics Society, American Academy of Orthopedic Surgeons Panel on Falls Prevention: Guideline for the prevention of falls in older persons. J Am Geriatr Soc 2001;49:664-672.

12 Liamis G, Milionis H, Elisaf M: A review of drug-induced hyponatremia. Am J Kidney Dis 2008;52:144-153.

13 Vaidya C, Ho W, Freda BJ: Management of hyponatremia: providing treatment and avoiding harm. Cleve Clin J Med 2010;77: 715-726.

14 Liamis G, Milionis H, Elisaf M: Blood pressure drug therapy and electrolyte disorders. Int J Clin Pract 2008;62:1572-1580.
15 Smith JM: Clinical implications of treating depressed older adults with SSRIs: possible risk of hyponatremia. J Gerontol Nurs 2010; $36: 22-27$

16 Mannesse CK, van Puijenbroek EP, Jansen PA, van Marum RJ, Souverein PC, Egberts TC: Hyponatremia as an adverse drug reaction of antipsychotic drugs: a case-control study in VigiBase. Drug Saf 2010;33:569578.

17 Buggy Y, Layton D, Fogg C, Shakir S: Safety profile of oxacarbazepine: results from a prescription-event monitoring study. Epilepsia 2010;51:818-829.

18 Dong X, Leppik I, White J: Hyponatremia from oxacarbazepine and carbamazepine. Neurology 2005;65;1976.

19 Tsiropolous I, Andersen M, Hallas J: Adverse events with use of antiepileptic drugs: a prescription and event symmetry analysis. Pharmacoepidemiol Drug Saf 2009; 18:483491.

20 Sarret D, Le Berre JP, Zemraoui N: Tramadol-induced hyponatremia. Am J Kidney Dis 2008;52:1026. 
21 Ellis G, Whitehead MA, Robinson D, O’Neill D, Langhorne P: Comprehensive geriatric assessment for older adults admitted to hospital: meta-analysis of randomized controlled trials. BMJ 2011;343:d6553.

22 Ellis G, Langhorne P: Comprehensive geriatric assessment for older hospital patients. $\mathrm{Br}$ Med Bull 2004;71:45-59.

23 Charlson ME, Pompei P, Ales KL, et al: A new method of classifying prognostic comorbidity in longitudinal studies: development and validation. J Chron Dis 1987;40: 373-383.

24 Salvi F, Miller MD, Grilli A, et al: A manual of guidelines to score the modified cumulative illness rating scale and its validation in acute hospitalized elderly patients. J Am Geriatr Soc 2008;56:1926-1931.

25 Hudon C, Fortin M, Soubhi H: Abbreviated guidelines for scoring the Cumulative Illness Rating Scale (CIRS) in family practice. J Clin Epidemiol 2007;60:212.

26 Talasz H, Jansen SC, Kofler M, Lechleitner M: High prevalence of pelvic muscle dysfunction in hospitalized elderly woman with urinary incontinence. Int Urogynecol J 2012, DOI 10.1007/s00192-011-1628-3.
27 Mahoney FI, Barthel DW: Functional evaluation: the Barthel index. Md State Med J 1965; 14:61-65.

28 Folstein MF, Folstein SE, McHugh PR, et al: 'Mini-mental state': a practical method for grading the cognitive status of patients for the clinicians. J Psychiatr Res 1975;12:189198.

29 Watson YI, Arfken CL, Birge SJ: Clock completion: an objective screening test for dementia. J Am Geriatr Soc 1993;41:12351240.

30 Warshw GA, Moore JT, Friedmann SW, et al: Functional disability in the hospitalized elderly. JAMA 1982;248:847-850.

31 Yesavage JA, Brink TL, Rose TL, et al: Development and validation of a geriatric depression screening scale: a preliminary report. J Psychiatr Res 1982;17:37-49.

32 Tinetti ME: Performance-oriented assessment of mobility problems in elderly patients. J Am Geriatr Soc 1986;34:119-126.

33 Podsiadlo D, Richardson S: The timed 'up and go': a test of basic functional mobility for frail elderly persons. J Am Geriatr Soc 1991; 39:142-148.

34 Guigoz Y, Garry JP: Mini nutritional assessment: a practical assessment tool for grading the nutritional state of elderly patients. Facts Res Gerontol 1994, pp 15-59.
35 Epstein M: Ageing and the kidney. J Am Soc Nephrol 1996;7:1106-1122.

36 Gianelli S, Patel K, Windham BG, et al: Magnitude of underascertainment of impaired kidney function in older adults with normal serum creatinine. J Am Geriatr Soc 2007;55: 816-823.

37 Rosholm JU, Nybo H, Anderson Ranberg K, Himmelstrup B, Skjelbo E, Christensen K, Gram LF: Hyponatremia in very old nonhospitalised people: association with drug use. Drugs Aging 2002;19:685-693.

38 Passare G, Viitanen M, Törring O, Winblad B, Fastbom J: Sodium and potassium disturbances in the elderly: prevalence and association with drug use. Clin Drug Invest 2004; 24:535-544.

39 Onder G, Petrovic M, Tangiisuran B, et al: Development and Validation of a Score to Assess Risk of Adverse Drug Reactions Among In-Hospital Patients 65 Years or Older: The GerontoNet ADR Risk Score Arch Intern Med 2010;170:1142-1148.

40 Greenberg A, Lehrich RW: Treatment of chronic hyponatremia: now we know how, but do we know when or if? J Am Soc Nephrol 2010;21:552-555. 